\title{
Craniofacial shape transition across the house mouse hybrid zone: implications for the genetic architecture and evolution of between-species differences
}

\author{
Luisa F. Pallares $^{1} \cdot$ Leslie M. Turner $^{1} \cdot$ Diethard Tautz $^{1}$
}

Received: 14 February 2016 / Accepted: 9 May 2016 / Published online: 23 May 2016

(C) The Author(s) 2016. This article is published with open access at Springerlink.com

\begin{abstract}
Craniofacial shape differences between taxa have often been linked to environmental adaptation, e.g., new food sources, or have been studied in the context of domestication. Evidence for the genetic basis of such phenotypic differences to date suggests that between-species as well as betweenpopulation variation has an oligogenic basis, i.e., few loci of large effect explain most of the variation. In mice, it has been shown that within-population craniofacial variation has a highly polygenic basis, but there are no data regarding the genetic basis of between-species differences in natural populations. Here, we address this question using a phenotypefocused approach. Using 3D geometric morphometrics, we phenotyped a panel of mice derived from a natural hybrid zone between Mus musculus domesticus and Mus mus musculus and quantify the transition of craniofacial shape along the hybridization gradient. We find a continuous shape transition along the hybridization gradient and unaltered developmental stability associated with hybridization. This suggests that the morphospace between the two subspecies is continuous despite reproductive isolation and strong barriers
\end{abstract}

Communicated by Nico Posnien and Nikola-Michael Prpic

This article is part of the Special Issue "Size and shape: integration of morphometrics, mathematical modelling, developmental and evolutionary biology," Guest Editors: Nico Posnien and Nikola-Michael Prpic.

Electronic supplementary material The online version of this article (doi:10.1007/s00427-016-0550-7) contains supplementary material, which is available to authorized users.

Diethard Tautz

tautz@evolbio.mpg.de

1 Department of Evolutionary Genetics, Max Planck Institute for Evolutionary Biology, August-Thienemannstr. 2,

24306 Plön, Germany to gene flow. We show that quantitative changes in overall genome composition generate quantitative changes in craniofacial shape; this supports a highly polygenic basis for between-species craniofacial differences in the house mouse. We discuss our findings in the context of oligogenic versus polygenic models of the genetic architecture of morphological traits.

Keywords Complex traits · Quantitative genetics · Skull shape $\cdot$ Mandible shape $\cdot$ Geometric morphometrics

\section{Introduction}

Data regarding the genetic basis of between-species differences have accumulated rapidly over the last two decades. In 1992, there were ten studies addressing "truly adaptive" traits between species pairs (Orr and Coyne 1992), i.e., the adaptive value of the trait was evident, like diapause timing in butterflies and flies. By 2001, 22 new studies had addressed the genetic basis of "ordinary" between-species differences (Orr 2001), i.e., differences that do not involve reduction of gene flow, in contrast with sterility/viability-related phenotypes (Orr 2001). Morphological differences are examples of this type of between-species differences. The latest and most extensive review of the loci underlying phenotypic differences, the so-called loci of evolution, includes 114 studies reporting between-species differences (Martin and Orgogozo 2013).

Sufficient data has now accumulated to enable informed discussion regarding the genetic nature of between-species differences. Long-standing questions include the role of small vs. large effect loci, regulatory vs. coding changes, single locus vs. many loci, and so forth. The data have shown that all such scenarios have occurred, and there is not a single or general way in which between-species differences have 
evolved (Orr 2001; Stern and Orgogozo 2008; Martin and Orgogozo 2013). However, the distribution of mutations causing phenotypic diversity seems to be non-random (Stern and Orgogozo 2009). Many examples of parallel genetic evolution show that the same gene or even the same nucleotide has been independently changed in different species/populations to generate the same type of adaptation (see examples in Stern and Orgogozo (2009)). This has suggested that the evolutionary process is to some extent predictable (Stern 2013).

Speciation geneticists have been particularly interested in finding the genetic basis of postzygotic barriers resulting in sterility or reduced viability in hybrids (Orr 2001; Wolf et al. 2010). Given the direct relevance such traits have in reproductive isolation between species, it is understandable why "ordinary" traits have often been of secondary interest. A good example of such "ordinary" differences is craniofacial morphology. This trait has been extensively studied from a developmental perspective; for example, there are 922 protein coding genes with reported craniofacial phenotypes in the mouse (Mouse Genome Informatics (MGI) database, queried on $14 / 01 / 16)$. However, the genes underlying natural variation in craniofacial traits between and within species are almost completely unknown. With the exception of Bmp3 in dogs (Schoenebeck et al. 2012), Ptchl in cichlids (Roberts et al. 2011), and five to six genes in Darwin's finches (Abzhanov et al. 2004, 2006; Mallarino et al. 2011; Lamichhaney et al. 2015), other data regarding between- or within-species craniofacial differences are limited to mapping studies where the causal genes underlying quantitative trait loci (QTL) still need to be identified.

The available mapping studies provide information about the genetic architecture of phenotypic differences. Results from QTL studies in fish, and dog breeds suggest that few loci of large effect explain most of the craniofacial differences between species/breeds (Albertson et al. 2003b; Kimmel et al. 2005; Boyko et al. 2010; Roberts et al. 2011; Schoenebeck et al. 2012). In contrast, studies of withinspecies variation in mice have shown the opposite picture, craniofacial variation is associated with many loci of small effect (Pallares et al. 2015). A discrepancy regarding the genetic architecture of within- and between-species variation has been reported for other traits (Orr 2001; Stern and Orgogozo 2008). However, in the particular case of craniofacial shape, such discrepancy is probably a consequence of the different evolutionary histories of the taxa, i.e., adaptive radiation/ artificial selection vs. weak (if any) selection. Using the same taxon to investigate within- and between-species variation might give more adequate insights.

The study of craniofacial variation in mice has been a very active field in the last decades. Several studies have explored craniofacial shape variation between subspecies of the house mouse (Mus musculus) around the world (Macholán 2006; Boell and Tautz 2011; Siahsarvie et al. 2012). The effect of hybridization on craniofacial traits has been studied using wild hybrid mice (Alibert et al. 1994; Auffray et al. 1996; Mikula and Macholán 2008; Mikula et al. 2010), as well as wildderived inbred lines representing different subspecies (Renaud et al. 2009, 2012). Our previous study mapping craniofacial traits in hybrid zone mice identified loci which potentially contribute to within- and/or between-subspecies variation (Pallares et al. 2014). However, to date, there are no studies addressing the genetic basis of natural craniofacial differences between the subspecies of the house mouse (but see Burgio et al. (2009); Burgio et al. (2012) for an approach using inbred lines of M. musculus and Mus spretus). In contrast, the genetic factors underlying differences between traditional inbred lines (i.e., LG/J, SM/J, A/J, and B6) have been extensively studied (Cheverud et al. 1997; Leamy et al. 1999; Klingenberg et al. 2004; Wolf et al. 2005; Burgio et al. 2009; Boell et al. 2011; Maga et al. 2015).

To address between-subspecies differences in a natural context, we take advantage of the house mouse European hybrid zone. This hybrid zone runs from Denmark to Bulgaria marking a climatic divide between Atlantic and Continental climate. Two subspecies of the house mouse, Mus musculus musculus and Mus musculus domesticus, have been in contact in this region for $\sim 3000$ years (reviewed in Baird and Macholán (2012)). We used first-generation labbred offspring of mice caught in the Bavarian region of the hybrid zone to quantify the transition of craniofacial shape along the hybridization gradient between $M . m$. musculus and $M$. m. domesticus.

We test the prediction that craniofacial shape differences between the two subspecies have a highly polygenic basis, i.e., shape differences are caused by many loci of small effect. If this is true, we expect that quantitative differences in the mean phenotype correspond to quantitative changes in mean genome composition, and therefore (1) the phenotypic transition should be continuous, (2) the mean shape at arbitrary points of the hybridization gradient should be different, and (3) shape changes between arbitrary points of the gradient should be directional.

\section{Methods}

\section{Mouse samples}

Wild hybrid mice were collected in the Bavarian region of the hybrid zone in Germany and brought to the Max Planck Institute for Evolutionary Biology in Plön, Germany (Turner et al. 2012). The mice were bred in the laboratory, and firstgeneration offspring were raised under standardized conditions. First-generation mice were sacrificed by $\mathrm{CO}_{2}$ asphyxiation between 9 and 13 weeks (see Turner et al. (2012) for details on animal experiments and ethics). Two hundred forty 
nine mice were used in this study; a subset of them was previously used in genome-wide association studies of craniofacial (Pallares et al. 2014) and sterility traits (Turner and Harr 2014). The sample used in this study includes siblings and half-siblings; details on the mice used in this study, including family information, are presented in Supplementary Table S1.

\section{Genotypes}

Mice were classified in nine hybrid groups based on genomic background, defined as the percentage of $M$. m. domesticus alleles (Table 1). Group 0 contains individuals with 0 to $9 \%$ M. m. domesticus alleles, and group 9 contains individuals with 90 to $100 \%$ M. m. domesticus alleles. The sample lacks mice with 70 to $79 \%$ of M. m. domesticus alleles, and therefore, hybrid group 7 was not analyzed in this study. The percentage of $M . m$. domesticus alleles was estimated by the average percentage of $M$. $m$. domesticus alleles of the parents. In a previous study, the parents were genotyped for 37 SNPs diagnostic of the two subspecies of house mouse, M. $\mathrm{m}$. musculus and M. m. domesticus (Turner et al. 2012). The group assignment based on average parent value was validated for a subset of mice using estimates of percentage $M . m$. domesticus alleles based on genotypes of 270 diagnostic autosomal SNPs (Turner et al. 2011). Group classifications based on estimates for individuals were highly correlated with classifications based on average parent values $(r=0.94$, $N=178$ ).

\section{Phenotypes}

Heads were scanned using a micro-computer tomograph microCT (vivaCT 40, Scanco, Bruettisellen, Switzerland). Energy and current were set to $70 \mathrm{kVp}$ and $177 \mu \mathrm{A}$; medium resolution was used generating a voxel size of $21 \mu \mathrm{m} .3 \mathrm{D}$ cross sections were reconstructed and imported into TINA tool (Schunke et al. 2012) where 44 3D-landmarks were located in the skull, and 13 3D-landmarks were located in each hemimandible.

A subset of 178 mice was previously phenotyped for craniofacial shape (Pallares et al. 2014). However, to ensure that all mice were phenotyped under the same conditions, all 249 mice used in this study were phenotyped in the following way: Landmarks were placed using the semi-automatic landmarking tool implemented in TINA tool (Bromiley et al. 2014) using a reference database of ten manually landmarked mice. Landmark position was revised and manually adjusted when necessary. Description of the landmarks used in this study can be found in Supplementary Table 2.

Raw landmark coordinates were exported to MorphoJ (Klingenberg 2011). Raw coordinates of right and left hemimandible were subjected to a generalized Procrustes analysis (GPA) where variation due to size, position, and location is removed, keeping only variation due to shape and allometry. The resulting Procrustes coordinates were averaged between right and left hemimandibles. Because the skull has object symmetry, raw coordinates were reflected and a GPA was done with the original and reflected datasets. Procrustes coordinates of original and reflected datasets were averaged per mouse. The average of right and left hemimandibles and original and reflected skulls represents a dataset that contains information only about the symmetric component of shape. The dimensionality of skull vectors is estimated by $\operatorname{dim}=3 k+$ $2 l-4$, where $k$ is the number of paired landmarks, and $l$ is the number of midline landmarks $(k=17, l=10, \operatorname{dim}=67)$ (Klingenberg et al. 2002). The dimensionality of mandible vectors is $\operatorname{dim}=3 k-7$, where $k$ is the number of landmarks $(k=13, \operatorname{dim}=32)$ (Klingenberg et al. 2002).

The asymmetric component of shape was not the main interest of this study; however, to explore developmental
Table 1 Hybrid individuals used in the analyses

\begin{tabular}{llllll}
\hline Hybrid group & \%Dom alleles & \%Dom alleles (average) & Families & Sex (m, f) & Sample size \\
\hline 0 & $0-9$ & 8.3 & 1 & 2,4 & 6 \\
1 & $10-19$ & 15.5 & 9 & 30,9 & 39 \\
2 & 24.3 & 17 & 67,0 & $67(65)$ \\
3 & $30-29$ & 7 & 23,0 & $23(22)$ \\
4 & $30-39$ & 5 & 12 & 17,14 & 31 \\
5 & $40-49$ & 43.1 & 10 & 25,0 & 26 \\
6 & $50-59$ & 55.2 & 3 & 3,6 & 31 \\
8 & $60-69$ & 62.3 & 7 & 11,6 & 9 \\
9 & $80-89$ & 89.4 & & Total sample size & $249(246)$ \\
\hline
\end{tabular}

Groups are defined based on the percentage of M. m. domesticus alleles (\%Dom alleles). The number of individuals, sex, number of families, and average percentage of $M . m$. domesticus alleles per group are shown. Where sample size differs between skull and mandible, skull sample size is indicated in parentheses 
stability along the hybridization gradient, a value of fluctuating asymmetry (FA) was generated for each individual using the method of Klingenberg and Monteiro (2005) implemented in MorphoJ. Each FA value represents the individual deviation from the mean FA of the group, in units of Procrustes distance.

Centroid size (CS) was calculated for each mouse skull using all landmarks (paired and midline). Since allometry accounted for $5.4 \%$ of total shape variation, and $23 \%$ of principal component 1 (PC1), its effect was excluded from the data using the residuals of a multivariate regression of shape on CS for further analyses. CS for mandible was calculated as the average of right and left hemimandibles. Allometry accounted for $1.8 \%$ of mandible shape variation and $6 \%$ of PC3. Its effect was excluded from the data following the same procedure used for skull. Differences in age have a negligible effect on shape variation $\left(r^{2}\right.$ (mandible $)=0.6 \%$, $p=0.08 ; r^{2}$ (skull) $\left.=0.7 \%, p=0.02\right)$. Sex differences account for 1.9 and $3.9 \%$ of shape variation in skull and mandible $(p<0.001)$, respectively, and $2 \%$ of size variation in skull and mandible $(p<0.02)$. Given that the sex effect is not strongly correlated with an individual PC (in contrast with allometric effects), and that females are well distributed among hybrid groups and do not form independent clusters from males (see Figs. 1 and 3), sexes were pooled within each group for further analysis.

Some of the individuals used here are siblings or halfsiblings (see Supplementary Table 1). However, all groups but one (group 0) are composed of more than one family (see Table 1), and therefore, because enough variation is available to make adequate inferences regarding between-group differences, related individuals were not pooled.

\section{Size variation}

Estimates of centroid size are not strongly affected by small sample size (Cardini and Elton 2007). Therefore, the mean CS of mandible and skull were calculated in MorphoJ for group 0 and 9 , and these values were used as proxy for M. m. musculus and M. m. domesticus size, respectively.

Fig. 1 Regression of centroid size (CS) on genome composition. Plot a shows data for skull CS and $\mathbf{b}$ for mandible CS. Males are shown in gray and females in open circles. Females do not cluster separately from males
To explore the variation in size across the hybrid gradient between M. m. musculus and M. m. domesticus, CS values were exported from MorphoJ into R (R-Core-Team 2015), and a linear regression of CS on hybrid group was performed. Pairwise Mann-Whitney tests were done for all pairwise comparisons of hybrid groups, and the results were adjusted for multiple testing using Holm-Bonferroni correction ( $\mathrm{R}$ function pairwise.wilcox.test).

\section{Shape differences between $M . m$. musculus and $M . m$. domesticus}

Groups 0 and 9 represent the extremes of the hybridization gradient, with an average of 8.3 and $91.8 \%$ M. m. domesticus alleles, respectively (Table 1). However, since the estimation of mean shape is very sensitive to small sample size (Cardini and Elton 2007), groups 0 and 1 (mus) and groups 8 and 9 (dom) were pooled to get more robust estimates of mean craniofacial shape in the extremes of the hybridization gradient.

The group mus contains 45 individuals with an average of $11.9 \%$ M. m. domesticus alleles ( $\min 8.3 \%$, max $18.3 \%$ ). The group dom contains 26 individuals with $90.6 \% \mathrm{M}$. $\mathrm{m}$. domesticus alleles on average ( $\min 88.3 \%, \max 99.3 \%)$. The mean shapes of $m u s$ and dom were generated in MorphoJ and were used as proxy for M. m. musculus and M. m. domesticus mean shapes, respectively.

The sample size of both groups together (71 individuals) is larger than mandible shape dimensionality $(\operatorname{dim}=32)$ and slightly larger than skull shape dimensionality $(\operatorname{dim}=67)$. However, to be consistent with the analyses performed with smaller sample sizes (see next section), we followed Evin et al. (2013) and Jombart et al. (2010) and reduced the dimensionality of shape data by performing a principal component analysis (PCA) using all 71 mice (R function prcomp) and retaining the first $10 \mathrm{PCs}$ representing $79 \%$ of the total phenotypic variation in mandible shape and $87 \%$ in skull shape.

The first 10 PCs for each trait were used to assess the differences in mean shape between mus and dom. The significance of the differences was determined with a two-sample
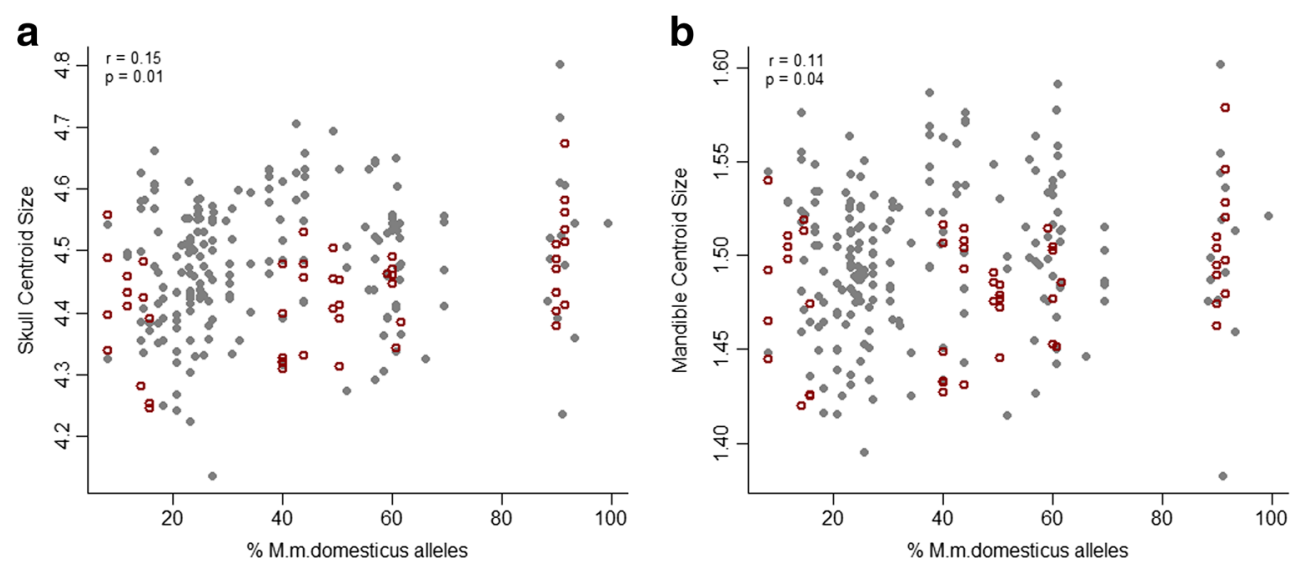
Hotelling's $T^{2}$ test ( $\mathrm{R}$ function HotellingsT2Test). The same PCs were used in a discriminant analysis (DA) to evaluate the separation between groups. The reliability of DA was assessed by a leave-one-out cross-validation procedure ( $\mathrm{R}$ function $l d a(C V=T))$.

\section{Shape variation along the hybrid gradient}

A multivariate regression of shape on percentage of $M . m$. domesticus alleles was used to evaluate whether skull and mandible shape were correlated with genomic admixture. The significance of the correlation was tested by permutation as implemented in MorphoJ; individuals assigned to each hybrid group were randomized without replacement and the sum of squares predicted by the regression was recorded; this procedure was repeated 10,000 times to create a null distribution. A univariate score was calculated for each mouse by projecting the shape vector of each individual onto the regression vector; in this way, a visual representation of the multivariate regression was generated.

As a second approach to explore shape transition along the hybrid gradient, a between-group PCA was performed using the group means (R function bga(type $=$ "pca")).

\section{Shape differences between hybrid groups}

With the aim of determining if changes in average genome composition result in distinct mean craniofacial shapes, skull and mandible mean shapes were compared between groups. This resulted in 36 comparisons. Sample size for four of these pairwise comparisons is smaller than mandible dimensionality $(\mathrm{dim}=32)$, and the sample size of 26 comparisons is smaller than skull shape dimensionality $(\mathrm{dim}=67)$. Therefore, we reduced the dimensionality of shape data as described above to be able to compare mean shapes. In short, following Evin et al. (2013) and Jombart et al. (2010), a PCA was performed for each pair of groups, and the first 10 PCs were retained and used in two-sample Hotelling's $T^{2}$ test and DA. DA was validated by the leave-one-out procedure. The total variance represented by the first 10 PCs ranged from 73 to $97 \%$ depending on the pair of groups (Supplementary Tables S3 and S4). The significance levels resulting from Hotelling's $T^{2}$ tests were adjusted for multiple testing using the Holm-Bonferroni method (R function p.adjust (method= "holm")).

Procrustes distance between groups was calculated in MorphoJ and represents shape distance. It is known that mean shape is sensitive to small sample size (Cardini and Elton 2007); therefore, the Procrustes distance between group means should be interpreted with caution when groups 0 $(N=6)$ and $8(N=9)$ are involved. Genomic distance between groups is estimated as the difference between mean percentage of M. m. domesticus alleles. The matrices of shape and genomic distance between groups were used to test for correlation between these metrics. For this purpose, a Mantel test was used, and the significance of the correlation was assessed by 10,000 permutation ( $\mathrm{R}$ function mantel.rtest).

Phenotypic variance per hybrid group was obtained in MorphoJ. It is calculated as the average of the sum of squared landmark deviations from the mean shape after Procrustes superimposition. Mean fluctuating asymmetry per group is calculated as the mean of FA per individual (see above, section "Phenotypes").

\section{Comparison of vector directions}

The angle between vectors can be used to assess the similarity between vector directions (Klingenberg and McIntyre 1998; Klingenberg and Marugan-Lobon 2013). To determine if the shape transition between groups is directional, i.e., whether it resembles the overall transition between $M$. m. musculus and $M$. $m$. domesticus, vectors between pairs of hybrid groups were calculated and compared to the vector going from mus (groups 0 and 1) mean shape to dom (groups 8 and 9) mean shape.

First, the vector of shape transition between hybrid groups was calculated such that the groups were always compared in ascending order in terms of the percentage of $M . m$. domesticus alleles. That is, the shape vector was constructed from the group with lower percentage to the group with higher percentage (e.g., group $0 \rightarrow$ group 1 , group $2 \rightarrow$ group 5).

Second, the vectors of shape transition between groups were compared to the vector representing shape changes from $m u s$ to dom. For this analysis, the angle between vectors was calculated and used to estimate the probability that the two vectors had the same direction due to chance. The assessment of significance was done using the formula from $\mathrm{Li}$ (2011) implemented in MorphoJ, and the resulting $p$ values were adjusted for multiple testing using the Holm-Bonferroni method.

\section{Results}

We have used 249 hybrid mice representing the hybridization continuum between M. m. musculus and M. m. domesticus to assess the transition of craniofacial shape and size between the two subspecies of the house mouse. With the aim of exploring in detail the nature of such transition, the mice were assigned to 9 hybrid groups according to the percentage of $M . m$. domesticus alleles (Table 1). The difference between consecutive hybrid groups is, on average, $10.4 \% \mathrm{M}$. m. domesticus alleles, with a minimum of $2.4 \%$ between groups 8 and 9 and a maximum of $27.1 \%$ between groups 6 and 8 .

\section{Craniofacial size}

There is a weak correlation between the percentage of $M . m$. domesticus alleles and skull CS $(r=0.15, p=0.01$, Fig. 1a) 
and mandible CS ( $r=0.11, p=0.04$, Fig. $1 b)$. However, none of the pairwise comparisons of CS between groups were significant after multiple testing correction, including the extremes of the hybridization gradient (groups 0 and 9).

\section{Shape differences in the extreme of the hybridization gradient}

Individuals from groups 0 and 1 ( $m u s$ ) and from groups 8 and 9 (dom) were combined (see "Methods") and used to estimate skull and mandible shape in the extremes of the hybridization gradient. The shape differences between mus and dom were significant for skull $\left(p=2 \times 10^{-16}, T^{2}(10,60)=65\right)$ as well as for mandible $\left(p=2 \times 10^{-26}, T^{2}(10,60)=55\right)$. Individuals representing $M . m$. domesticus are characterized by a straighter mandible compared to the mean mus mandible; this is visible by the relative arrangement of the condyle, coronoid, and angular processes (Fig. 2b). The lower molar row of mus is shorter and more distant from the body of the mandible. Mus individuals have also a shorter alveolar ramus but a higher ascending ramus and a more pronounced angular process relative to the condyle (Fig. 2a-c). M. m. musculus is characterized by a wider and higher rostrum relative to the back of the skull, shorter frontal bone relative to the nasal and parietal bones, and an upper molar row shifted towards the interior of the mouth, while individuals representing $M . m$. domesticus have a straight molar row (Fig. 2d-g).

The Procrustes distance between mean skull shapes is 0.026 , and 0.042 between mean mandible shapes ( $p(1000$ perm) $<0.0001$ ). The leave-one-out cross-validation procedure correctly assigned all individuals to their original group.

\section{Shape transition along the hybridization gradient}

Skull and mandible shape is significantly correlated with the relative genome composition of the individuals. A multivariate regression of shape on percent $M . m$. domesticus alleles showed that $8.4 \%$ of skull shape variation $(p(10,000$ perm $)=0.0001)$ and $10.8 \%$ of mandible shape variation $(p(10,000$ perm $)=<0.0001)$ can be explained by average genome composition (Fig. 3). Moreover, the shape transition along the hybridization gradient is continuous, with overlaps between individual shapes found in each group, resulting in no major gaps between groups.

Continuous variation is also reflected in the strong positive correlation between Procrustes distance and genomic distance between groups (skull: $r=0.78, p=1 \times 10^{-4}$; mandible: a
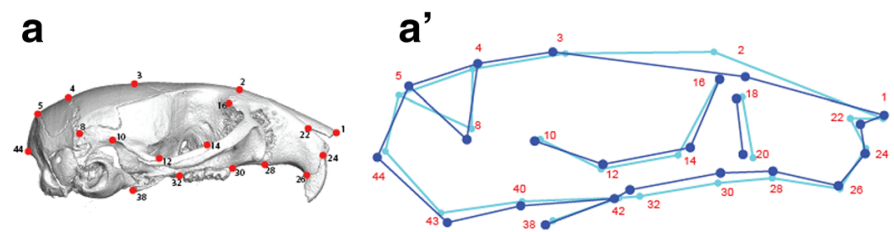

C
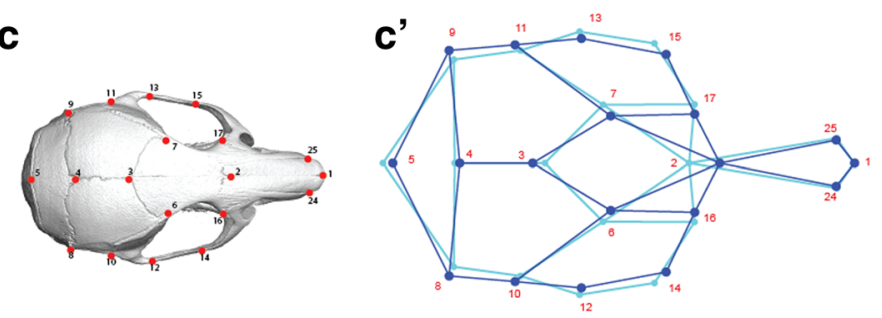

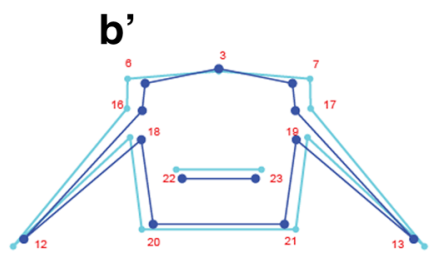

b

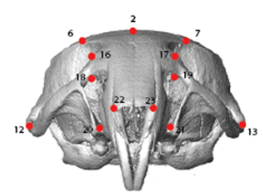

d'

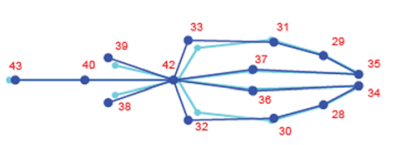

d

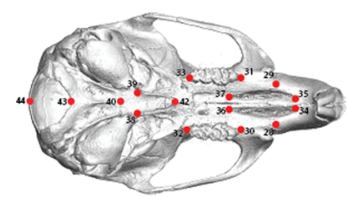

e

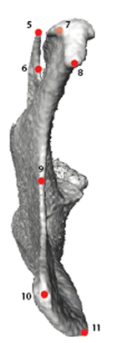

d'

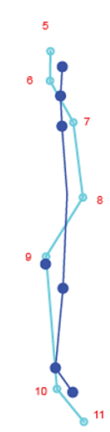

f'

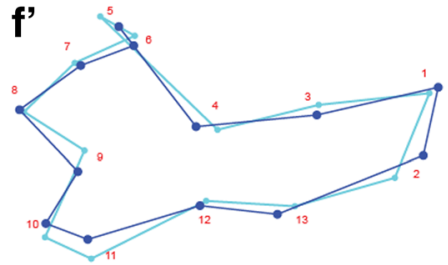

g'

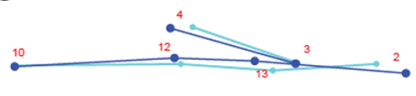

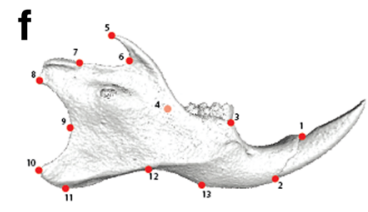

g

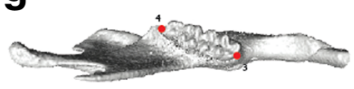

Fig. 2 Skull and mandible shape changes from mus to dom. Images of skull and mandible are shown $(a-g)$ together with the landmarks representative of each view. 2D wireframes $\left(a^{\prime}-g^{\prime}\right)$ are used to represent the 3D shape changes from mus mean shape (light blue) to dom mean shape (dark blue). Landmarks are numbered and represented as dots. Lateral view $\left(a, a^{\prime}, f, f^{\prime}\right)$, frontal view $\left(b, b^{\prime}, e, e^{\prime}\right)$, dorsal view $\left(c, c^{\prime}, g\right.$, $\left.g^{\prime}\right)$, and skull ventral view $\left(d, d^{\prime}\right)$. Shape changes are magnified $\times 3$ 
a

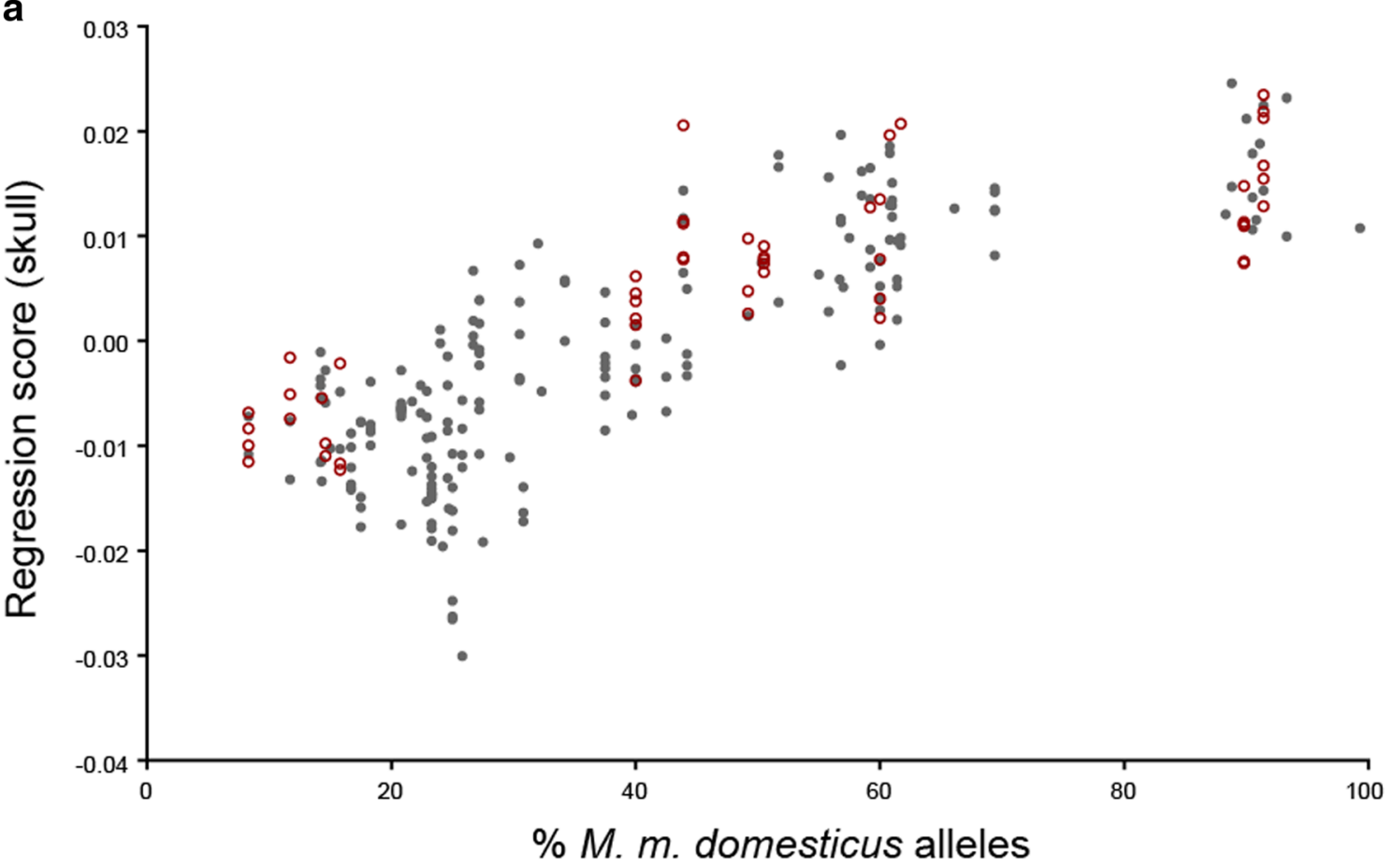

b

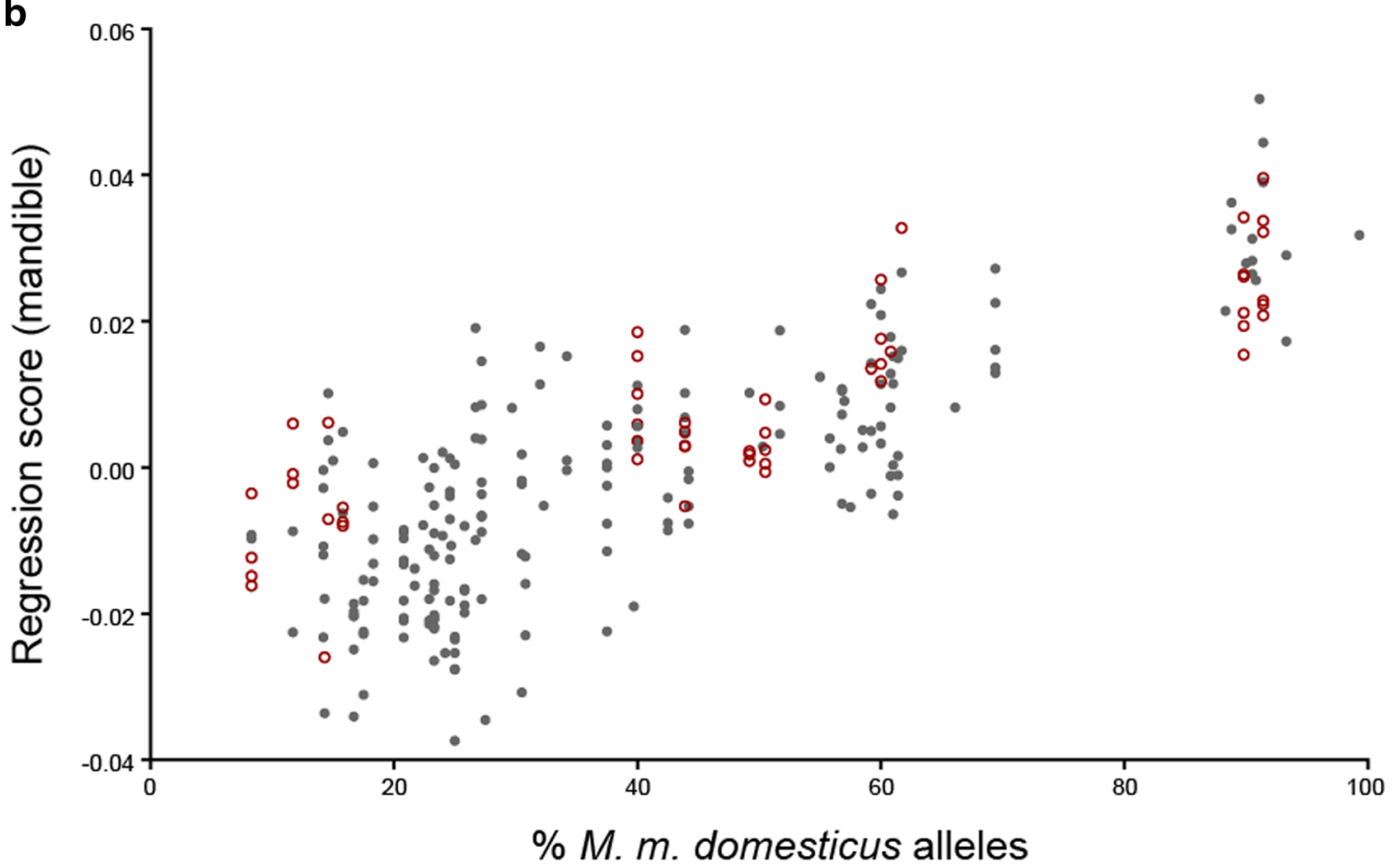

Fig. 3 Multivariate regression of shape on genome composition. Plot a shows data for skull and $\mathbf{b}$ for mandible. For the purpose of visualization, a univariate score was generated for each mouse. Each point represents the projection of an individual shape vector onto the vector derived from

$r=0.92, p=1 \times 10^{-4}$ ) - the larger the genomic distance, the larger the phenotypic distance (Fig. 4a, b). However, for pairs of groups with genomic distance less than $20 \%$, the correlation is no longer significant (Fig. 4c, d). the multivariate regression (see "Methods"). Males are shown in gray and females in open circles. Skull $p(10,000$ permutations $)<0.0001$, $r^{2}=8.4 \%$. Mandible $p(10,000$ permutation $)<0.0001, r^{2}=10.8 \%$

Phenotypic variance differs between groups, but it is not correlated with the degree of hybridization $(p($ skull $)=0.72 ; p$ $($ mand $)=0.93)($ Fig. 5). However, it is correlated with the number of families per group $(r=0.64, p=0.036)$; groups 

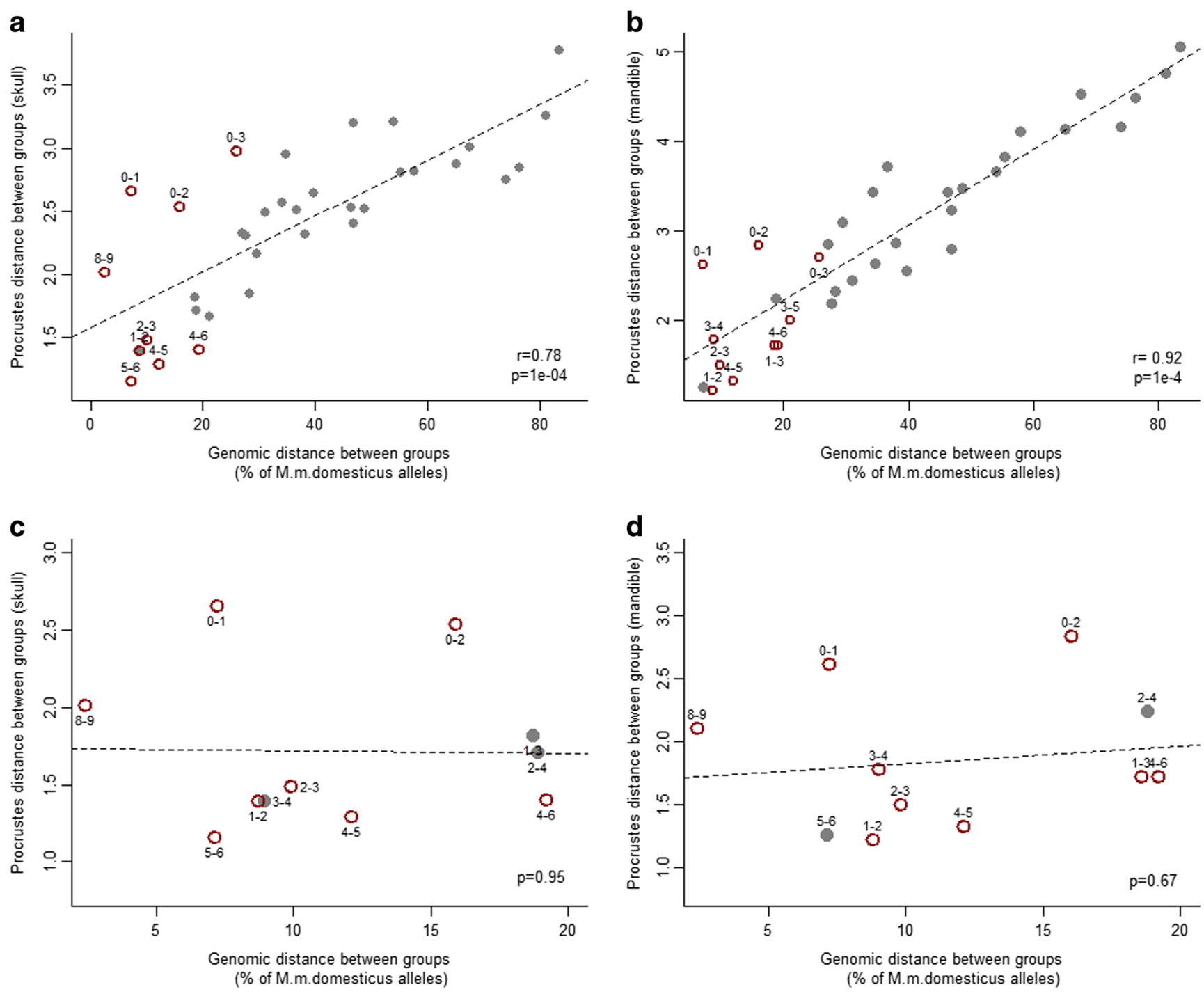

Fig. 4 Correlation between shape distance and genomic distance. Procrustes distance between group mean shapes is used as a measure of shape distance. The difference between mean percentage of $M . m$. domesticus alleles is used as proxy for genomic distance. Each point represents a pairwise comparison between groups. All comparisons are shown for skull (a) and for mandible (b). Pairs of groups with a genomic distance smaller than $20 \%$ are shown for skull (c) and for mandible (d).

with more unrelated individuals tend to have higher phenotypic variance. Skull variance ranges from 0.020 to 0.031 units of Procrustes distance, while mandible variance ranges from 0.026 to 0.040 . Despite these marked changes in phenotypic variance, levels of fluctuating asymmetry remain constant across groups (Fig. 5); FA in skull and mandible is, on average, 0.01 and 0.028 units of Procrustes distance, respectively.

\section{Shape differences between hybrid groups}

Differences in skull and mandible mean shape were assessed for all pairs of hybrid groups. All group means differ significantly from each other, except for groups 8 and $9(p$ (skull) $=0.21$,

Open circles are pairwise comparisons that do not follow the mus-to-dom directionality (see Table 3). Procrustes and genomic distances between each pair of groups can be found in supplementary Tables S3 and S4. A Mantel test was used to test for correlation between Procrustes and genomic distance matrices. The significance was derived by 10,000 permutations

$p($ mandible $)=0.07)($ Table 2 and Supplementary Table S5), which showed small genomic and Procrustes distances (Fig. 4).

Figure 6 shows the ordination of the mean skull and mandible shape of all hybrid groups. A continuous transition is recovered in the first two principal component axes, with a clear gap where group 7 would likely be located if there were individuals with such genome composition in the sample of mice used here.

\section{Direction of shape changes between groups}

Shape change vectors were calculated between each pair of groups and compared with the shape change vector 
Fig. 5 Phenotypic variation and developmental stability along the hybridization gradient. Plot a shows data for skull and $\mathbf{b}$ for mandible. Fluctuating asymmetry $(F A)$ is used as a proxy for developmental stability. FA values for individual mice are shown in blue dots, and the blue line indicates the mean FA value per group. Individual FA values are in units of Procrustes distance from the mean FA of the group. Red dots show the phenotypic variation per group, calculated as the squared root of the sum of squared landmark deviations from the group mean shape
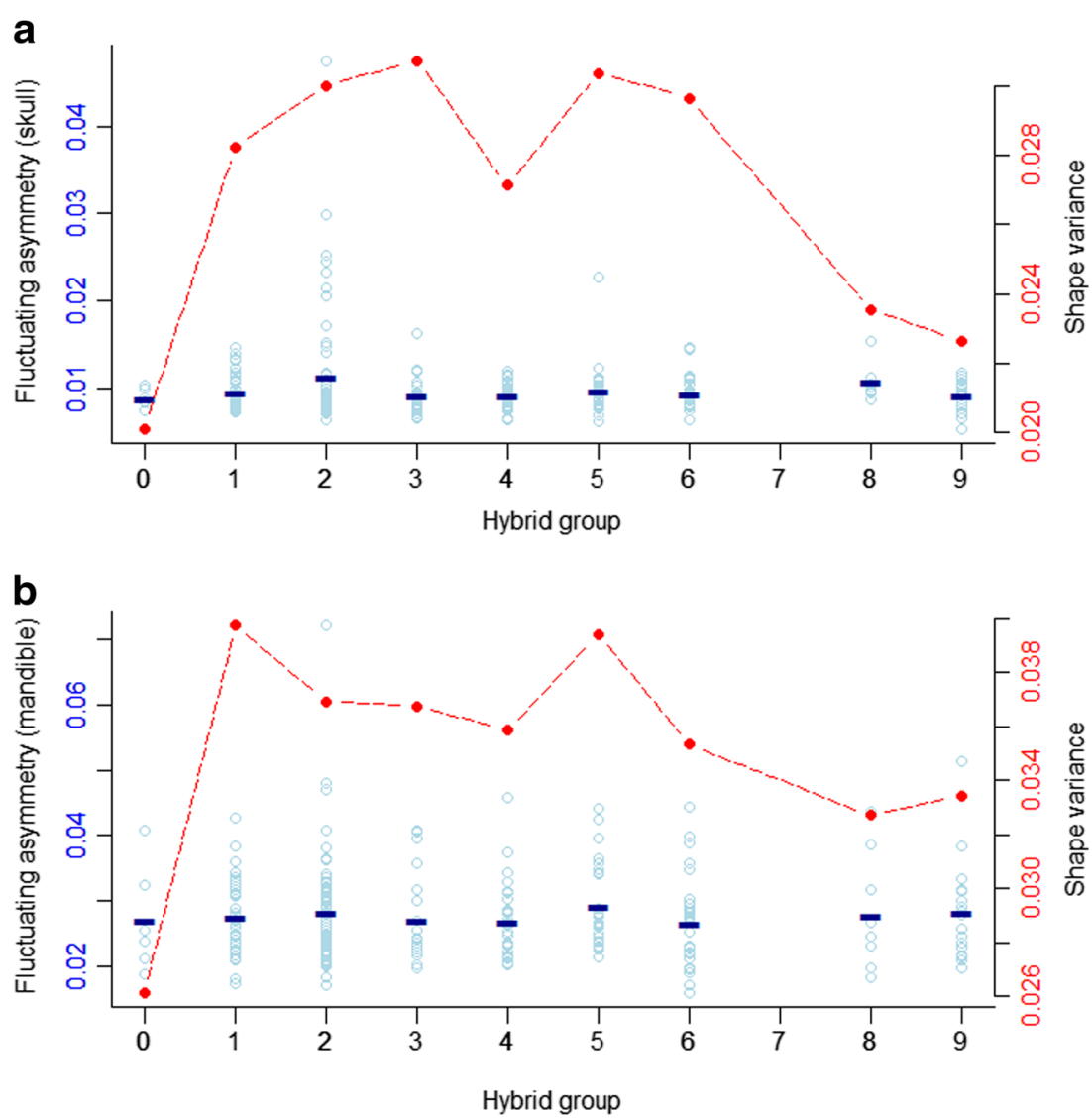

from mus (groups 0 and 1) to dom (groups 8 and 9). After multiple testing correction, 27 out of 36 pairs (75\%) are consistent with the mus-to-dom skull transition and 25 pairs $(69 \%)$ are consistent with the mus-to-dom mandible transition (Table 3). Pair of groups that do not show consistent directions of change in shape space are predominantly those with small genomic distances (average distance $13.6 \%$ M. m. domesticus alleles), regardless of the Procrustes distance between them (Fig. 4a, b).

\section{Discussion}

We have explored the transition of craniofacial shape and size along a hybridization gradient between two subspecies of the house mouse, $M . m$. musculus and $M . m$. domesticus. The mice used in this study are derived from wild-caught hybrids and are thus representative of natural phenotypic and genotypic variation across the hybrid zone. At the same time, it is a sample with enhanced genetic effects relative to environmental effects because mice were raised in controlled laboratory conditions. With this design, we controlled for non-genetic factors affecting shape variation yet analyzed variation within an evolutionarily relevant context.

\section{Craniofacial size}

There are no significant differences in skull or mandible size between hybrid groups. The lack of differentiation between groups 0 and 9 , at the extremes of the hybridization gradient indicates that $M . m$. musculus and M. m. domesticus do not differ in craniofacial size. Comparisons between wild-derived mouse strains representing the same two subspecies found the same pattern for mandible (WLA and PWK) (Renaud et al. 2009, 2012) and for skull (DDO and MDH) (Debat et al. 2000). However, differences in molar size have been reported between strains DDO and MDH (Alibert et al. 1997).

Heterosis of mouse mandible (Renaud et al. 2009), skull (Debat et al. 2000; Percival et al. 2015), and molar size (Alibert et al. 1997) has been reported previously, with F1 individuals being larger than the parental lines. The mice used here do not include F1 or F2 individuals, because there were no F1 hybrids sampled in the hybrid zone (Turner et al. 2012). Therefore, the lack of size differentiation between groups in the extreme of the hybrid gradient and the other groups indicates that any size heterosis that might have been present in the first generations of intercross between the subspecies was lost in later generations. Results from crosses between WLA and PWK inbred strains suggest that the heterosis present in F1 is lost as soon as the second generation (Renaud et al. 2012). 
Table 2 Mean shape comparisons between hybrid groups

\begin{tabular}{|c|c|c|c|c|c|c|c|c|c|}
\hline Skull & 0 & 1 & 2 & 3 & 4 & 5 & 6 & 8 & 9 \\
\hline 0 & & $9 \%$ & $1 \%$ & $0 \%$ & $0 \%$ & $6 \%$ & $0 \%$ & $7 \%$ & $4 \%$ \\
\hline 1 & $4 \mathrm{E}-05$ & & $15 \%$ & $10 \%$ & $9 \%$ & $5 \%$ & $0 \%$ & $0 \%$ & $0 \%$ \\
\hline 2 & $3 \mathrm{E}-09$ & $2 \mathrm{E}-11$ & & $11 \%$ & $2 \%$ & $1 \%$ & $2 \%$ & $0 \%$ & $0 \%$ \\
\hline 3 & $2 \mathrm{E}-06$ & $3 \mathrm{E}-09$ & $5 \mathrm{E}-09$ & & $6 \%$ & $8 \%$ & $4 \%$ & $0 \%$ & $0 \%$ \\
\hline 4 & $3 \mathrm{E}-09$ & $5 \mathrm{E}-14$ & $5 \mathrm{E}-24$ & $2 \mathrm{E}-09$ & & $14 \%$ & $18 \%$ & $3 \%$ & $2 \%$ \\
\hline 5 & $5 \mathrm{E}-06$ & $4 \mathrm{E}-17$ & $4 \mathrm{E}-26$ & $3 \mathrm{E}-07$ & $2 \mathrm{E}-06$ & & $19 \%$ & $0 \%$ & $0 \%$ \\
\hline 6 & $6 \mathrm{E}-10$ & $4 \mathrm{E}-20$ & $9 \mathrm{E}-32$ & $3 \mathrm{E}-13$ & $5 \mathrm{E}-08$ & $1 \mathrm{E}-04$ & & $5 \%$ & $4 \%$ \\
\hline 8 & $7 \mathrm{E}-08$ & $3 \mathrm{E}-13$ & $9 \mathrm{E}-20$ & $2 \mathrm{E}-09$ & $6 \mathrm{E}-08$ & $6 \mathrm{E}-09$ & $1 \mathrm{E}-06$ & & $27 \%$ \\
\hline 9 & $3 \mathrm{E}-08$ & $9 \mathrm{E}-20$ & $6 \mathrm{E}-29$ & $1 \mathrm{E}-12$ & $5 \mathrm{E}-12$ & $1 \mathrm{E}-14$ & $3 \mathrm{E}-09$ & 0.21 & \\
\hline Mandible & 0 & 1 & 2 & 3 & 4 & 5 & 6 & 8 & 9 \\
\hline 0 & & $7 \%$ & $1 \%$ & $3 \%$ & $0 \%$ & $6 \%$ & $3 \%$ & $7 \%$ & $0 \%$ \\
\hline 1 & $6 \mathrm{E}-03$ & & $21 \%$ & $13 \%$ & $10 \%$ & $5 \%$ & $3 \%$ & $2 \%$ & $0 \%$ \\
\hline 2 & $1 \mathrm{E}-06$ & $5 \mathrm{E}-08$ & & $23 \%$ & $11 \%$ & $13 \%$ & $4 \%$ & $1 \%$ & $0 \%$ \\
\hline 3 & $9 \mathrm{E}-05$ & $4 \mathrm{E}-08$ & $2 \mathrm{E}-04$ & & $19 \%$ & $16 \%$ & $11 \%$ & $0 \%$ & $0 \%$ \\
\hline 4 & $2 \mathrm{E}-09$ & $1 \mathrm{E}-11$ & $2 \mathrm{E}-14$ & $2 \mathrm{E}-04$ & & $33 \%$ & $13 \%$ & $0 \%$ & $0 \%$ \\
\hline 5 & $9 \mathrm{E}-06$ & $9 \mathrm{E}-13$ & $7 \mathrm{E}-14$ & $1 \mathrm{E}-04$ & 0.038 & & $28 \%$ & $0 \%$ & $0 \%$ \\
\hline 6 & $4 \mathrm{E}-07$ & $2 \mathrm{E}-14$ & $2 \mathrm{E}-20$ & $1 \mathrm{E}-07$ & $6 \mathrm{E}-06$ & 0.025 & & $3 \%$ & $6 \%$ \\
\hline 8 & $5 \mathrm{E}-05$ & $6 \mathrm{E}-15$ & $1 \mathrm{E}-16$ & $4 \mathrm{E}-08$ & $2 \mathrm{E}-09$ & $1 \mathrm{E}-07$ & $1 \mathrm{E}-07$ & & $35 \%$ \\
\hline 9 & $2 \mathrm{E}-05$ & $6 \mathrm{E}-16$ & $3 \mathrm{E}-23$ & $4 \mathrm{E}-11$ & $2 \mathrm{E}-14$ & $5 \mathrm{E}-11$ & $3 \mathrm{E}-08$ & $\mathbf{0 . 0 6 9}$ & \\
\hline
\end{tabular}

Skull and mandible results are shown. The lower triangle (dark green) shows the $\mathrm{p}$ value resulting from two-sample Hotelling's $T^{2}$ test after multiple testing correction. The upper triangle (light green) shows the percentage of misclassified individuals derived from a linear discriminant analysis using a leave-one-out cross-validation procedure. In red are the pairwise comparisons for groups with undistinguishable mean shape. See Supplementary Table $\mathrm{S} 5$ for $T^{2}$ statistics and degrees of freedom

\section{Craniofacial shape}

In contrast with craniofacial size, skull and mandible shapes are clearly different between $M . m$. musculus (group mus) and M. m. domesticus (group dom). The separation between groups is perfect, even when the mice representing each subspecies have on average $\sim 10 \%$ alleles coming from the other subspecies. Given such clear differences between the extremes of the hybridization gradient, it is of particular interest to ask how such traits change with the percentage of $M . m$. domesticus alleles in the genome. When visually inspected, the transition along the hybridization gradient seems continuous, without major gaps between hybrid groups (Fig. 3). Such a continuous pattern was first observed in a sample of wild mice collected in the Danish hybrid zone between $M . m$. musculus and M. m. domesticus (Auffray et al. 1996). In that study, the ventral side of the skull was phenotyped using 2D geometric morphometrics. Here, we have explored the entire skull using 3D geometric morphometrics, confirming the results from Auffray et al. (1996), and have expanded the

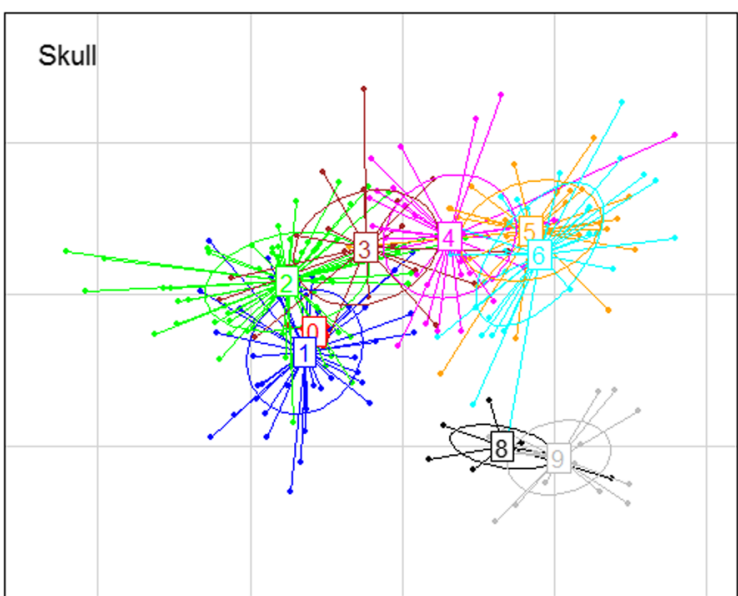

Fig. 6 Between-group principal component analysis. The plot represents the ordination of mean skull shape and mean mandible shape of the hybrid groups (squares). Dots are individuals projected onto the PC

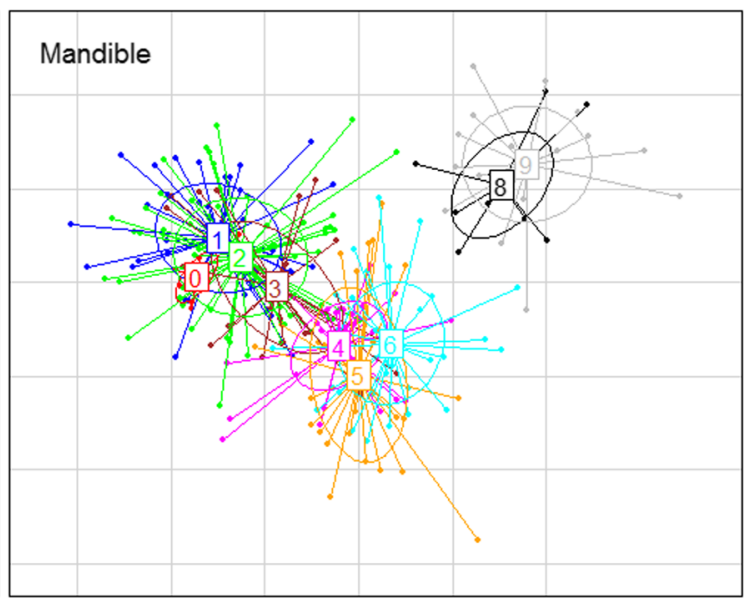

axes defined by the mean shape ordination. Each hybrid group is represented by a different color. The numbers inside the boxes represent the groups 
Table 3 Comparison of vector directions

\begin{tabular}{|c|c|c|c|c|c|c|c|c|c|}
\hline Skull & 0 & 1 & 2 & 3 & 4 & 5 & 6 & 8 & 9 \\
\hline 0 & & $\mathbf{0 . 8 8}$ & $\mathbf{0 . 8 8}$ & $\mathbf{0 . 2 8}$ & $4 \mathrm{E}-03$ & $4 \mathrm{E}-04$ & $4 \mathrm{E}-04$ & $4 \mathrm{E}-04$ & $4 \mathrm{E}-04$ \\
\hline 1 & $90.3^{\circ}$ & & $\mathbf{0 . 8 3}$ & 0.041 & $4 \mathrm{E}-04$ & $4 \mathrm{E}-04$ & $4 \mathrm{E}-04$ & $4 \mathrm{E}-04$ & $4 \mathrm{E}-04$ \\
\hline 2 & $87.2^{\circ}$ & $84.2^{\circ}$ & & $\mathbf{0 . 0 5 3}$ & $4 \mathrm{E}-04$ & $4 \mathrm{E}-04$ & $4 \mathrm{E}-04$ & $4 \mathrm{E}-04$ & $4 \mathrm{E}-04$ \\
\hline 3 & $78.8^{\circ}$ & $71.0^{\circ}$ & $72.3^{\circ}$ & & $9 \mathrm{E}-04$ & $4 \mathrm{E}-04$ & $4 \mathrm{E}-04$ & $4 \mathrm{E}-04$ & $4 \mathrm{E}-04$ \\
\hline 4 & $65.9^{\circ}$ & $58.1^{\circ}$ & $50.9^{\circ}$ & $63.3^{\circ}$ & & $\mathbf{0 . 1 9}$ & $\mathbf{0 . 1 0}$ & $1 \mathrm{E}-03$ & $4 \mathrm{E}-04$ \\
\hline 5 & $61.8^{\circ}$ & $54.8^{\circ}$ & $56.3^{\circ}$ & $56.1^{\circ}$ & $76.4^{\circ}$ & & $\mathbf{0 . 8 8}$ & 0.044 & $4 \mathrm{E}-04$ \\
\hline 6 & $60.3^{\circ}$ & $48.2^{\circ}$ & $50.8^{\circ}$ & $57.0^{\circ}$ & $74.2^{\circ}$ & $86.2^{\circ}$ & & 0.044 & $4 \mathrm{E}-04$ \\
\hline 8 & $44.4^{\circ}$ & $31.5^{\circ}$ & $39.9^{\circ}$ & $51.4^{\circ}$ & $63.6^{\circ}$ & $71.4^{\circ}$ & $71.4^{\circ}$ & & $\mathbf{0 . 2 7}$ \\
\hline 9 & $43.3^{\circ}$ & $13.6^{\circ}$ & $29.3^{\circ}$ & $39.6^{\circ}$ & $52.2^{\circ}$ & $60.4^{\circ}$ & $57.6^{\circ}$ & $78.0^{\circ}$ & \\
\hline Mandible & 0 & 1 & 2 & 3 & 4 & 5 & 6 & 8 & 9 \\
\hline 0 & & 1 & $\mathbf{1}$ & $\mathbf{0 . 4 2}$ & 0.013 & 0.047 & $3 \mathrm{E}-03$ & $4 \mathrm{E}-04$ & $4 \mathrm{E}-04$ \\
\hline 1 & $89.1^{\circ}$ & & $\mathbf{0 . 9 9}$ & $\mathbf{0 . 1 2}$ & $2 \mathrm{E}-03$ & $6 \mathrm{E}-03$ & $4 \mathrm{E}-04$ & $4 \mathrm{E}-04$ & $4 \mathrm{E}-04$ \\
\hline 2 & $86.1^{\circ}$ & $83.0^{\circ}$ & & $\mathbf{0 . 1 7}$ & 0.012 & 0.013 & $4 \mathrm{E}-04$ & $4 \mathrm{E}-04$ & $4 \mathrm{E}-04$ \\
\hline 3 & $74.8^{\circ}$ & $67.0^{\circ}$ & $69.8^{\circ}$ & & $\mathbf{0 . 1 3}$ & $\mathbf{0 . 1 3}$ & $2 \mathrm{E}-03$ & $4 \mathrm{E}-04$ & $4 \mathrm{E}-04$ \\
\hline 4 & $58.5^{\circ}$ & $52.3^{\circ}$ & $58.0^{\circ}$ & $67.9^{\circ}$ & & $\mathbf{1}$ & $\mathbf{0 . 0 6 3}$ & $4 \mathrm{E}-04$ & $4 \mathrm{E}-04$ \\
\hline 5 & $63.0^{\circ}$ & $56.0^{\circ}$ & $58.6^{\circ}$ & $67.7^{\circ}$ & $86.2^{\circ}$ & & 0.012 & $4 \mathrm{E}-04$ & $4 \mathrm{E}-04$ \\
\hline 6 & $54.5^{\circ}$ & $41.7^{\circ}$ & $47.5^{\circ}$ & $52.5^{\circ}$ & $64.2^{\circ}$ & $58.2^{\circ}$ & & $6 \mathrm{E}-04$ & $4 \mathrm{E}-04$ \\
\hline 8 & $33.4^{\circ}$ & $19.4^{\circ}$ & $24.2^{\circ}$ & $31.7^{\circ}$ & $41.0^{\circ}$ & $43.2^{\circ}$ & $50.0^{\circ}$ & & $\mathbf{0 . 4 9}$ \\
\hline 9 & $28.2^{\circ}$ & $10.2^{\circ}$ & $19.6^{\circ}$ & $24.1^{\circ}$ & $27.6^{\circ}$ & $36.7^{\circ}$ & $41.2^{\circ}$ & $76.6^{\circ}$ & \\
\hline
\end{tabular}

The vector of shape change between groups was compared to the vector of shape change between mus and dom. Results for skull and mandible are shown. The lower triangle (dark green) shows the angle in degrees between the two vectors. The upper triangle (light green) shows the significance of the correlation between vectors after correction for multiple testing. In red are the pairs of groups whose transition along the mus-to-dom direction is not significantly supported

analysis to the mandible, finding a similar pattern of continuous variation.

From a developmental point of view, such continual variation indicates that all craniofacial shapes derived from the random combination of the two genomes are realizable and therefore that the shape space between $M$. m. musculus and $M$. $m$. domesticus is continuous. The level of fluctuating asymmetry is constant along the hybridization gradient even when phenotypic variance changes across groups; this indicates that the degree of hybridization does not affect the developmental stability of the phenotype (Fig. 4). However, results from wild-caught animals suggest that the morphological cline for ventral skull shape may be steeper than the change in hybrid index and therefore that some impairment in skull development could be associated with certain hybrid genotypes when environmental effects and selection in the wild are into play (Auffray et al. 1996). So far, there is no formal analysis of craniofacial morphology clines in the house mouse hybrid zone. This will be necessary to estimate gene by environment effects and determine if selection is acting against some specific craniofacial morphologies.

\section{Shape differences between hybrid groups}

A more detailed exploration of the shape transition along the hybridization gradient showed that each one of the nine hybrid groups defined based on the percentage of $M$. m. domesticus alleles has a different mean craniofacial shape, except for groups 8 and 9 that differ neither in skull nor in mandible shape. The pairwise genomic distance between groups that differ in mean shape ranges from 7.1 to $83.5 \% \mathrm{M}$. $\mathrm{m}$. domesticus alleles, indicating that even small changes in genome composition generate quantifiable changes in the phenotype. It should be noted that the presence of related individuals reduces phenotypic variation within groups (see "Results"); however, all but one group are composed of more than one family (see Table 1), and therefore, differences in mean shape between groups cannot not be explained by family effects.

Studies of consomic lines between C57BL/6 and PWD strains (Boell et al. 2011), and interspecific congenic strains between C57BL/6 and SEG/Pas (Burgio et al. 2009, 2012), have also shown that small differences in genome composition can generate quantifiable changes in the phenotype. However, the results from Boell et al. (2011) and Burgio et al. (2009, 2012) should be interpreted from the perspective of the individual, as inbred lines represent a single point in the universe of possible genotypes. The use of wild or outbred mice, like the sample used here, has the advantage of providing a more accurate picture of between-individual variation, because many combinations of the loci relevant for craniofacial shape are likely to be present within the same hybrid group. It therefore provides an understanding of the dynamics of the phenotype from a population perspective. Results from 
inbred lines showed that craniofacial shape is responsive to changes in specific loci; by contrast, our results indicate that shape is also responsive to the cumulative number of alleles at causal loci in the population.

While quantitative changes in genome composition from one group to the next generate distinct craniofacial shapes, the direction of change is conserved. Most of the pairwise comparisons between groups are consistent with differences between $M . m$. musculus and M. m. domesticus. This indicates that a directional walk in shape space is possible regardless of the specific loci that are being added or removed in each step. On the contrary, what seems to matter is the quantitative composition of the genome.

\section{Implications for the genetic basis of between-species craniofacial differences}

Taken together, our results suggest that many loci of small effect determine the craniofacial differences between $M . m$. musculus and M. m. domesticus. The shape differences between subspecies are caused by different alleles at these loci, but the interactions among them appear predominantly additive since they produce developmentally stable phenotypes in the hybrids; this is evident from the constant values of fluctuating asymmetry along the hybridization gradient. Further, the smooth transition between the subspecies excludes the possibility that few loci of large effect are responsible for betweenspecies differences.

By contrast, loci of large effect have been implicated in craniofacial differences in other taxa. Between 23 and 46 QTLs have been associated with craniofacial differences between species of cichlids, with individual QTL explaining up to $52 \%$ of phenotypic variation (Albertson et al. 2003a, b). Later, it was shown that Ptchl is the causal gene underlying a large effect locus for lower jaw differences between cichlid species (Roberts et al. 2011). A study of morphological variation in dogs explored 55 traits including many craniofacial measurements; the top three SNPs per trait explained on average $67 \%$ of phenotypic variation between breeds (Boyko et al. 2010). A different study showed that five QTL explain most of the skull variation between dog breeds and identified $B m p 3$ as one of the underlying causal genes (Schoenebeck et al. 2012). Different stickleback ecotypes have very characteristic craniofacial shapes, one to five QTL have been found to explain these differences (Kimmel et al. 2005). Genes associated with beak variation in Darwin's finches have qualitatively large effects; however, their effect still needs to be quantified in terms of between-species variation.

Although it is known that mapping studies overestimate the absolute phenotypic effect of each QTL (Beavis 1998), the overall message from these studies is that relatively few loci are responsible for between-species and between-population craniofacial variation, which seems to be in contrast with our results. However, there is a major difference between these studies and ours with respect to the history of adaptation of the corresponding taxa. In the fish and dog studies, craniofacial diversity is associated with strong selection during adaptive radiation and domestication, respectively. Under such scenarios, it has been predicted that phenotypic differences are generated by an exponential distribution of mutational effects (Orr 1998), where few mutations of large effect generate an initial rapid change in the phenotype that later is refined by mutations of small effect.

Major adaptive changes in morphology are not evident in the house mouse system. Although some specific adaptations appear to occur when new habitats or islands are invaded (Renaud and Auffray 2010; Boell and Tautz 2011; Renaud et al. 2013; Babiker and Tautz 2015), these remain subtle. Hence, the divergence between separated mouse populations and subspecies may be equally influenced by neutral accumulation of allelic differences. Under this scenario, we do not expect to see loci of large effect underlying betweensubspecies differences. However, the relative importance of drift and selection still needs to be quantified.

Using a GWAS approach, we have previously shown that the genetic architecture of within-population craniofacial shape variation in the mouse is highly polygenic (Pallares et al. 2015). In this study, using a phenotype-focused approach, we find patterns that support a highly polygenic basis for between-subspecies differences. Different experimental approaches (GWAS, congenic lines, consomic panels) using different type of mice (inbred, outbred, wild) have found a positive correlation between the length of genomic fragments and the magnitude of their effect on craniofacial shape (Burgio et al. 2009, 2012; Boell et al. 2011; Pallares et al. 2014, 2015). Here we also find a strong positive correlation between genomic distance and phenotypic distance. Therefore, the evidence seems strong enough to suggest that, irrespective of the taxonomic level at which differences are being observed, craniofacial shape variation in the mouse has a highly polygenic basis.

\section{Conclusions}

The mice used here are representative of a wild population where diploid organisms are expected to have a unique allelic composition relative to each other. Making use of this characteristic, we have shown that the highly polygenic architecture of shape determination results in a situation in which more or less any mixture of alleles generates a developmentally stable phenotype with smooth transitions between different degrees of admixture. This implies that in cases of micro-evolutionary adaptation to environmental changes, many genes could be involved in modifying the shape in the required direction. 
Acknowledgments We thank Sabrina Renaud for fruitful discussions and Bettina Harr for sharing mouse samples. This work was supported by institutional funds of the Max Planck Society to DT and funds from the Deutsche Forschungsgemeinschaft to B. Harr (SFB-680). This project was developed while LFP was part of the International Max Planck Research School (IMPRS) for Evolutionary Biology. Open access funding provided by Max Planck Society.

Open Access This article is distributed under the terms of the Creative Commons Attribution 4.0 International License (http:// creativecommons.org/licenses/by/4.0/), which permits unrestricted use, distribution, and reproduction in any medium, provided you give appropriate credit to the original author(s) and the source, provide a link to the Creative Commons license, and indicate if changes were made.

\section{References}

Abzhanov A, Protas M et al (2004) Bmp4 and morphological variation of beaks in Darwin's finches. Science 305(5689):1462-1465

Abzhanov A, Kuo WP et al (2006) The calmodulin pathway and evolution of elongated beak morphology in Darwin's finches. Nature 442(7102):563-567

Albertson RC, Streelman JT et al (2003a) Directional selection has shaped the oral jaws of Lake Malawi cichlid fishes. Proc Natl Acad Sci U S A 100(9):5252-5257

Albertson RC, Streelman JT et al (2003b) Genetic basis of adaptive shape differences in the cichlid head. J Hered 94(4):291-301

Alibert P, Renaud S et al (1994) Fluctuating asymmetry in the mus musculus hybrid zone: a heterotic effect in disrupted co-adapted genomes. Proc Biol Sci 258(1351):53-59

Alibert P, Fel-Clair F et al (1997) Developmental stability, fitness, and trait size in laboratory hybrids between European subspecies of the house mouse. Evolution 51(4):1284-1295

Auffray JC, Alibert P et al (1996) Relative warp analysis of skull shape across the hybrid zone of the house mouse (Mus musculus) in Denmark. J Zool 240:441-455

Babiker H, Tautz D (2015) Molecular and phenotypic distinction of the very recently evolved insular subspecies Mus musculus helgolandicus Zimmermann, 1953. BMC Evol Biol 15(1):1-14

Baird SJE, Macholán M (2012) What can the Mus musculus musculus/ M. m. domesticus hybrid zone tell us about speciation? In: Macholán M, Baird SJE, Munclinger P, Pialek J (eds) Evolution of the house mouse. Cambridge University Press, Cambridge, pp 334 372

Beavis WD (1998) QTL analyses: power, precision, and accuracy. In: Paterson A (ed) Molecular analysis of complex traits. CRC Press, Boca Raton, pp 145-161

Boell L, Tautz D (2011) Micro-evolutionary divergence patterns of mandible shapes in wild house mouse (Mus musculus) populations. BMC Evol Biol 11:306

Boell L, Gregorova S et al (2011) A comparative assessment of mandible shape in a consomic strain panel of the house mouse (Mus musculus)-implications for epistasis and evolvability of quantitative traits. BMC Evol Biol 11:309

Boyko AR, Quignon P et al (2010) A simple genetic architecture underlies morphological variation in dogs. PLoS Biol 8(8), e1000451

Bromiley PA, Schunke AC et al (2014) Semi-automatic landmark point annotation for geometric morphometrics. Front Zool 11:61

Burgio G, Baylac M et al (2009) Genetic analysis of skull shape variation and morphological integration in the mouse using interspecific recombinant congenic strains between $\mathrm{C} 57 \mathrm{BL} / 6$ and mice of the mus spretus species. Evolution 63(10):2668-2686

Burgio G, Baylac M et al (2012) Exploration of the genetic organization of morphological modularity on the mouse mandible using a set of interspecific recombinant congenic strains between $\mathrm{C} 57 \mathrm{BL} / 6$ and mice of the Mus spretus species. G3 2:1257-1268

Cardini A, Elton S (2007) Sample size and sampling error in geometric morphometric studies of size and shape. Zoomorphology 126(2): 121-134

Cheverud JM, Routman EJ et al (1997) Pleiotropic effects of individual gene loci on mandibular morphology. Evolution 51(6):2006-2016

Debat V, Alibert P et al (2000) Independence between developmental stability and canalization in the skull of the house mouse. Proc R Soc B Biol Sci 267(1442):423-430

Evin A, Cucchi T et al (2013) The long and winding road: identifying pig domestication through molar size and shape. J Archaeol Sci 40(1): 735-743

Jombart T, Devillard S et al (2010) Discriminant analysis of principal components: a new method for the analysis of genetically structured populations. BMC Genet 11:94

Kimmel CB, Ullmann B et al (2005) Evolution and development of facial bone morphology in threespine sticklebacks. Proc Natl Acad Sci U S A 102(16):5791-5796

Klingenberg CP (2011) MorphoJ: an integrated software package for geometric morphometrics. Mol Ecol Resour 11(2):353-357

Klingenberg CP, Marugan-Lobon J (2013) Evolutionary covariation in geometric morphometric data: analyzing integration, modularity, and allometry in a phylogenetic context. Syst Biol 62(4):591-610

Klingenberg CP, McIntyre GS (1998) Geometric morphometrics of developmental instability: analyzing patterns of fluctuating asymmetry with Procrustes methods. Evolution 52:1363-1375

Klingenberg CP, Monteiro LR (2005) Distances and directions in multidimensional shape spaces: implications for morphometric applications. Syst Biol 54(4):678-688

Klingenberg CP, Barluenga $\mathrm{M}$ et al (2002) Shape analysis of symmetric structures: quantifying variation among individuals and asymmetry. Evolution 56(10):1909-1920

Klingenberg CP, Leamy LJ et al (2004) Integration and modularity of quantitative trait locus effects on geometric shape in the mouse mandible. Genetics 166:1909-1921

Lamichhaney S, Berglund J et al (2015) Evolution of Darwin/'s finches and their beaks revealed by genome sequencing. Nature 518(7539): 371-375

Leamy LJ, Routman EJ et al (1999) Quantitative trait loci for early- and late-development skull characters in mice: a test of the genetic independence model of morphological integration. Am Nat 153:201214

Li S (2011) Concise formulas for the area and volume of a hyperspherical cap. Asian J Math Stat 4:66-70

Macholán M (2006) A geometric morphometric analysis of the shape of the first upper molar in mice of the genus Mus (Muridae, Rodentia). J Zool 270(4):672-681

Maga AM, Navarro N et al (2015) Quantitative trait loci affecting the 3D skull shape and size in mouse and prioritization of candidate genes in-silico. Front Physiol 6:92

Mallarino R, Grant PR et al (2011) Two developmental modules establish 3D beak-shape variation in Darwin's finches. Proc Natl Acad Sci U S A 108(10):4057-4062

Martin A, Orgogozo V (2013) The loci of repeated evolution: a catalog of genetic hotspots of phenotypic variation. Evolution 67(5):12351250

Mikula O, Macholán M (2008) There is no heterotic effect upon developmental stability in the ventral side of the skull within the house mouse hybrid zone. J Evol Biol 21(4):1055-1067 
Mikula O, Auffray J-C et al (2010) Asymmetric size and shape variation in the Central European transect across the house mouse hybrid zone. Biol J Linn Soc 101(1):13-27

Orr HA (1998) The population genetics of adaptation: the distribution of factors fixed during adaptive evolution. Evolution 52:935-949

Orr HA (2001) The genetics of species differences. Trends Ecol Evol 16(7):343-350

Orr HA, Coyne JA (1992) The genetics of adaptation: a reassessment. Am Nat 140(5):725-742

Pallares LF, Harr B et al (2014) Use of a natural hybrid zone for genomewide association mapping of craniofacial traits in the house mouse. Mol Ecol 23:5756-5770

Pallares LF, Carbonetto P et al (2015) Mapping of craniofacial traits in outbred mice identifies major developmental genes involved in shape determination. PLoS Genet 11(11), e1005607

Percival CJ, Liberton DK et al. (2015) Genetics of murine craniofacial morphology: diallel analysis of the eight founders of the Collaborative Cross. J Anat

R-Core-Team (2015) R: a language and environment for statistical computing. R Foundation for Statistical Computing, Vienna

Renaud S, Auffray JC (2010) Adaptation and plasticity in insular evolution of the house mouse mandible. J Zool Syst Evol Res 48(2):138-150

Renaud S, Alibert P et al (2009) Mandible shape in hybrid mice. Naturwissenschaften 96(9):1043-1050

Renaud S, Alibert P et al (2012) Modularity as a source of new morphological variation in the mandible of hybrid mice. BMC Evol Biol 12(1471-2148 (Electronic)):141

Renaud S, Hardouin EA et al (2013) Invasive house mice facing a changing environment on the Sub-Antarctic Guillou Island (Kerguelen Archipelago). J Evol Biol 26(3):612-624
Roberts RB, Hu Y et al (2011) Craniofacial divergence and ongoing adaptation via the hedgehog pathway. Proc Natl Acad Sci U S A 108(32):13194-13199

Schoenebeck JJ, Hutchinson SA et al (2012) Variation of BMP3 contributes to dog breed skull diversity. PLoS Genet 8(8), e1002849

Schunke A, Bromiley P et al (2012) TINA manual landmarking tool: software for the precise digitization of 3D landmarks. Front Zool 9(1):6

Siahsarvie R, Auffray J-C et al (2012) Patterns of morphological evolution in the mandible of the house mouse Mus musculus (Rodentia: Muridae). Biol J Linn Soc 105(3):635-647

Stern DL (2013) The genetic causes of convergent evolution. Nat Rev Genet 14(11):751-764

Stern DL, Orgogozo V (2008) The loci of evolution: how predictable is genetic evolution? Evolution 62(9):2155-2177

Stern DL, Orgogozo V (2009) Is genetic evolution predictable? Science 323(5915):746-751

Turner LM, Harr B (2014) Genome-wide mapping in a house mouse hybrid zone reveals hybrid sterility loci and Dobzhansky-Muller interactions. Elife 3

Turner LM, Schwahn DJ et al. (2011) Data from: reduced male fertility is common but highly variable in form and severity in a natural house mouse hybrid zone, Dryad Data Repository

Turner LM, Schwahn DJ et al (2012) Reduced male fertility is common but highly variable in form and severity in a natural house mouse hybrid zone. Evolution 66(2):443-458

Wolf JB, Leamy LJ et al (2005) Epistatic pleiotropy and the genetic architecture of covariation within early and late-developing skull trait complexes in mice. Genetics 171(2):683-694

Wolf JBW, Lindell J et al (2010) Speciation genetics: current status and evolving approaches. Philos Trans R Soc Lond B 365(1547):17171733 